\title{
Guidelines for antimicrobial utilization in health care facilities
}

Health Services Directorate, Health Services and Promotion Branch: and Bureau of Communicable Disease epidemiology, Health Protection Branch, HeAlth and Welfare CANADA, OTtaWA, Ontario

\section{PREAMBLE}

Inappropriate use of antimicrobial agents within health care facilities is a frequent occurrence, with documented prevalence rates as high as $40 \%$. The consequences of inappropriate antimicrobial use are major and include development of resistance, acquisition of nosocomial infections, drug toxicity and increased cost. Both the physician and the institution have a responsibility to provide optimal and cost-effective patient care, as well as to minimize adverse drug effects. The following guidelines are formulated to assist health care facilities in developing and implementing institutional programs to promote appropriate antimicrobial use. Emphasis is placed on the general principles of antimicrobial use, the essential components of an institutional antimicrobial use program, and the organization and implementation of such a program.

Members of the working group on Antimicrobial Utilization in Health Care Facilities were: Anthony Chow. MD.

(Chairperson) Vancouver, British Columbia; R Elder, MD. Ottawa, Ontario: Peter Jewesson, PhD, Vancouver, British Columbia; Lionel Lavoie, MD, Melfort, Saskatchewan; Lionel Mandell, MD. Hamilton. Ontario: Lindsay Nicolle, MD. Winnipeg, Manitoba: Ori Rotstein, MD, Toronto, Ontario; Walter Schlech, MD, Halifax, Nova Scotia: Marjorie Carroll, RN, MEd, Ottawa, Ontario; Jeanne Casault-Seguin, Ottawa, Ontario

Published by Health Services and Promotion Branch. Department of National Health and Welfare, 1990

Reproduced with permission of the Minister of Supply and Services Canada, 1990

\section{ANTIMICROBIAL USE}

\section{General principles}

Antimicrobials should only be used for specific indications: ie, either treatment of documented infection, treatment of suspected infection, or prophylaxis of infection.

Justification for and choice of antimicrobial agents should be based on scientifically validated clinical studies wherever possible.

Choice of antimicrobial agents should be made according to known or suspected pathogens, knowledge of local susceptibility patterns, and pharmacokinetic attributes of the agents. In general, agents with proven efficacy and the most narrow antimicrobial spectrum should be chosen. Clinical efficacy should be the primary consideration. A secondary consideration is toxicity. When efficacy and toxicity issues are equivalent, cost considerations become a priority.

Sound pharmacologic principles should be considered in establishing the appropriate usage of antimicrobial agents.

The effects of antimicrobial use/misuse should be evaluated not only for the individual patient, but also for the potential impact on the institution and the population at large.

\section{Indications}

Antimicrobial drugs are used either for prophylactic or therapeutic indications. 


\section{Prophylactic indications}

Antimicrobials are used prophylactically to prevent infections from occurring, as opposed to using them to treat established infection. To use these drugs optimally, they should be given so that the drug is present at the potential site of infection, in appropriate concentrations, when the patient is at risk from the infecting pathogen. Their use should ideally be limited to those situations in which there is adequate proof of efficacy. In some situations, such proof may not exist, but the medical consensus is that the risk of infection warrants chemoprophylaxis.

Drugs can be used for prophylaxis in medical or surgical settings.

Chemoprophylaxis for medical conditions may be indicated either to prevent conditions associated with specific organisms, eg, meningitis due to meningococcus, or to prevent selected clinical syndromes associated with a variety of pathogens, such as bacterial endocarditis. Refer to the Annotated Bibliography for a more detailed description. Chemoprophylaxis for surgical settings is aimed at the prevention of postoperative wound infection. Patents selected to receive prophylactic antimicrobials should, in general, be those who are at high risk for postoperative wound infection (ie, clean-contaminated operations) or patients in whom wound infections are associated with high morbidity and mortality rates (ie, insertion of prosthetic material). Having defined the patient population to receive prophylactic antimicrobial agents, several guidelines should be used to govern their use. These include:

- The antimicrobial agent chosen should be directed against the pathogens most commonly responsible for wound infection at the particular surgical site.

- Adequate tissue levels of the antimicrobial agent should be achieved during the surgical procedure itself. As such, administration of the prophylactic agent should occur within 30 mins to $1 \mathrm{~h}$ before the start of the operative procedure, for instance at induction of anesthesia. If an antimicrobial agent with a short half-life is used, additional intraoperative doses may be required to maintain adequate tissue levels if the operation is delayed or prolonged. In certain circumstances there may be exceptions. For instance, in Caesarian sections a prophylactic agent may be given at the time of cord clamping.

- The duration of postoperative prophylactic antimicrobial use is generally short. Use of antimicrobials beyond $24 \mathrm{~h}$ is usually not in- dicated. Local hospital practice will help define this issue. Extended use of antimicrobial prophylaxis is not associated with increased efficacy but may increase the risk of drug toxicity, superinfection and changes in the patient's flora.

More detailed information concerning antimicrobial prophylaxis, including recommended regimens, in the surgical patient can be obtained in the references provided in the Annotated Bibliography.

\section{Therapeutic indications}

Antimicrobials are used for therapeutic purposes when a clinically important infection is suspected or documented. Not all infections require antimicrobial therapy. The therapeutic use of antimicrobials can be either empiric or specific.

Antimicrobials are used empirically for those situations in which infection is strongly suspected on clinical grounds but microbiologic confirmation is not yet available.

The following factors should be considered when using antimicrobials for empiric treatment of infection:

- likely site(s) of infection and the most probable pathogen(s)

- knowledge of susceptibility patterns of bacteria in the hospital

- host factors, eg, neutropenia, renal dysfunction, etc

- modification of the initial regimen when additional clinical and laboratory data are available, usually at 48 to $72 \mathrm{~h}$.

Antimicrobials are used for specific or nonempirical indications when the infecting microorganisms are known.

\section{Duration of therapy}

Few definite guidelines exist as to the optimal duration of therapy. As a very general rule, antimicrobials should be used empirically for approximately 48 to $72 \mathrm{~h}$ and then re-evaluated if necessary according to clinical response and culture and sensitivity results. In the event that infection is not proven microbiologically, but strong clinical suspicion of infection remains, empiric therapy should be continued until a clinical response is seen and the patient is afebrile for several days.

In most instances, a proven infection is treated for approximately seven to 10 days provided that there is a good response. However, certain infections may be treated for shorter periods of time, 
while others may require longer periods of therapy.

\section{THE INSTITUTIONAL ANTIMICROBIAL USE PROGRAM}

The health care facility has a responsibility to ensure appropriate, safe and cost-effective antimicrobial therapy. This goal appears to be most readily achieved by establishing a multidisciplinary antimicrobial use program, usually through the Antimicrobial Use Subcommittee of the Institutional Pharmacy and Therapeutics Committee. The responsibilities of this committee are:

- to identify potential problem areas of antimicrobial use by developing databases through an antimicrobial surveillance program

- to effectively transmit antimicrobial use information to hospital and medical staff

- to implement educational and control measures designed to optimize antimicrobial use.

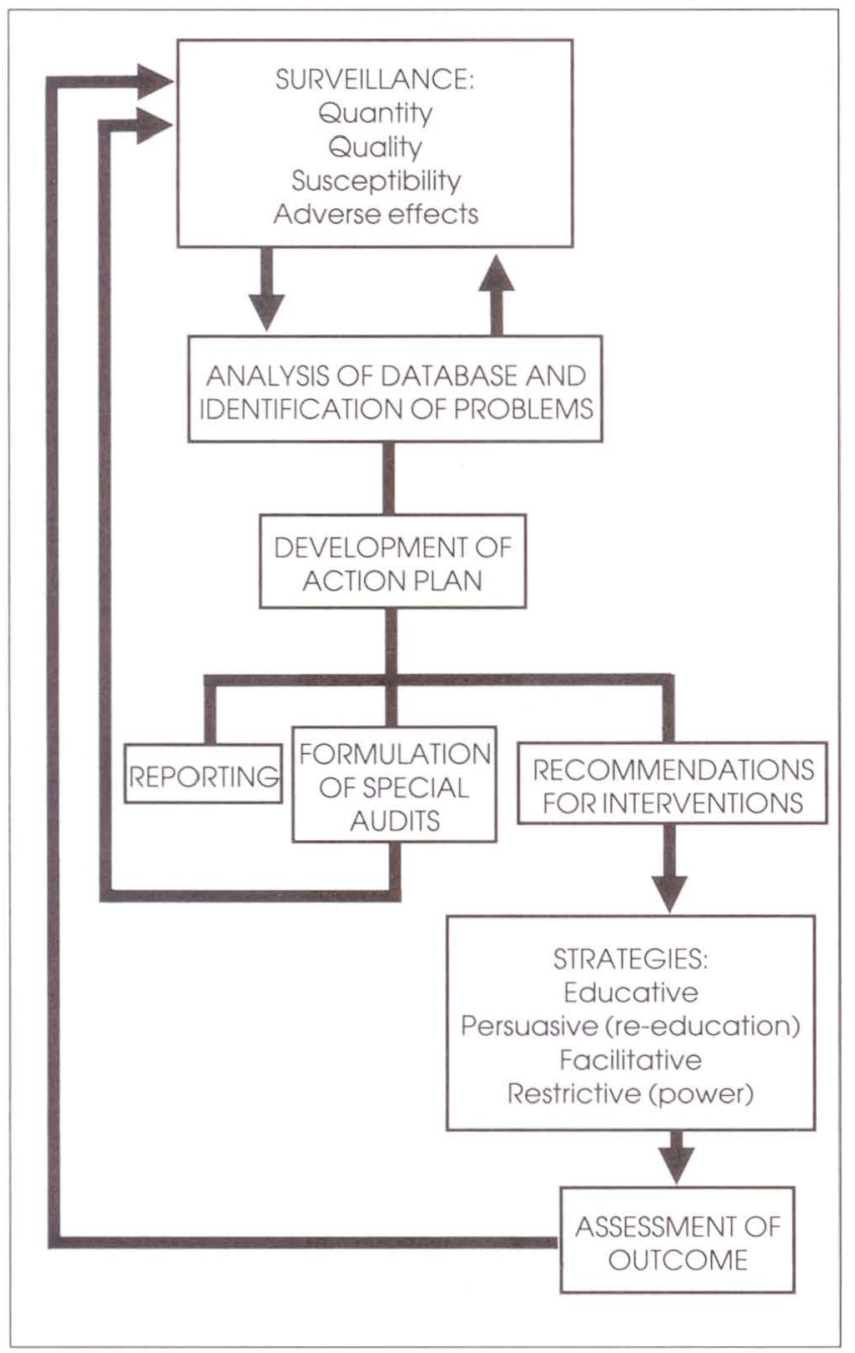

\section{Organization and implementation}

- To ensure the success of all facets of an antimicrobial use program, it is essential that this committee has the support of the medical staff and the endorsement, as well as authorization, of the highest level of hospital administration.

- It is essential that an appropriate budget and necessary resources, including designated personnel, are made available for the implementation and efficient operation of this program. The savings associated with appropriate antimicrobial utilization policies within the hospital are substantial. Cost savings realized should be documented, and a proportion of savings should be redirected to sustain the program.

- To achieve maximal impact, the committee should ensure and facilitate meaningful interaction among various segments within the institution, such as nursing, pharmacy, microbiology, infectious diseases clinicians and other medical staff, medical records, quality assurance, cost containment and infection control committees.

\section{ESSENTIAL COMPONENTS OF THE AN- TIMICROBIAL USE PROGRAM}

\section{Identification of the problems of antimicrobial use through surveillance and database development}

Effective antimicrobial use programs require that a mechanism be established to survey antimicrobial use and identify potential problem areas for more detailed investigation.

The specific aims of an antimicrobial use surveillance program are:

- to develop the antimicrobial use database for periodic review and analysis

- to identify potential problem areas and select the most important of these for usage review

- to report and disseminate information to administration and medical staff for appropriate action.

The illustration (left) outlines the essential components of an antimicrobial use program.

\section{Surveillance activities}

The following surveillance activities are recommended for database development:

- quantity of drug use 
- quality of drug use

- antimicrobial susceptibility profiles

- adverse effects of drugs.

Data collection for database development may be performed in several ways. Surveillance may be prospective or retrospective, active or passive, and may be comprehensive or based on random sampling. Representatives from several different areas of the hospital should be involved in developing the structure of the database, gathering data and analyzing the outcome of the audit. They should include hospital pharmacy, medical staff, microbiology, infection control, quality assurance and hospital administration.

\section{Quantity of use (antimicrobial utilization)}

Methods of surveillance which are available and may be complementary are:

- Pharmacy purchase orders. These may provide information concerning ordering trends, usage volumes and expenditure. However, these records are probably too crude to use for deriving detailed information regarding antimicrobial use within a given time frame.

- Drug distribution records. Within the institution these may more accurately reflect current drug use. This method is particularly accurate in hospitals using the unit dose system.

- Unit dose system. This lessens the inaccuracies associated with stockpiling certain drugs on the patient-care unit over a prolonged period of time and reduces wastage.

- Antimicrobial order sheet. The most accurate and comprehensive method of monitoring antimicrobial utilization is through the introduction of an antimicrobial order sheet. Although effective, this approach is labour-intensive and requires the cooperation of the medical staff.

\section{Quality of use (antimicrobial usage)}

The most important aspect of evaluating the quality of antimicrobial usage is the application of pre-established criteria which define the appropriateness of use. Such guidelines should not only define optimal usage of an antimicrobial as it relates to dosing, interval and toxicity monitoring, but should also predetermine the threshold of inappropriate usage when corrective action should be taken. These criteria should adhere to both published guidelines and local practice. At a given institution, the criteria should be estab- lished through the consensus of medical staff in consultation with infectious disease and microbiology specialists.

Having established criteria, activities should focus on monitoring adherence and exceptions to policy. The types of activities include:

- Antimicrobial usage audit. This includes monitoring a specific antimicrobial or family of antimicrobials which might be prone to excessive or inappropriate use, or are very toxic or expensive.

- Site- or organism-specific infections. Antimicrobial therapy for a specific disease entity or a particular microorganism would be assessed in this form of audit.

- Service-specific cross-sectional antimicrobial usage. This audit would review drug usage in defined patient areas, such as in the Intensive Care Unit or on the Surgical Service.

\section{Susceptibility profiles}

The Microbiology Department has a central role in reporting antimicrobial susceptibility patterns to the Antimicrobial Use Subcommittee, the Infection Control Committee, the Pharmacy and Therapeutics Committee and individual clinical departments, as warranted. While the susceptibility pattern of a specific microorganism is important for a given patient, knowledge of the overall prevalence and changing trends in susceptibility patterns within the facility is critical for developing institutional recommendations of antimicrobial therapy.

The microbiology laboratory should review susceptibility data periodically, as determined by the Antimicrobial Use Subcommittee, and report summaries of microorganism identification and susceptibility patterns with appropriate cross tabulations. Potentially significant alterations in trends should be reported when identified. Minimal requirements for this database include:

- microorganism

- site

- service

- patient identifiers (eg, patient identification number, etc).

Adverse effects of antimicrobial use The following are methods for identifying potentially adverse effects of antimicrobial use.

- Critical incident reporting. Most hospitals have in place a mechanism for identifying severe drug reactions through critical incident report- 
ing. However, minor adverse reactions are unlikely to be identified by this method.

- Monitoring of serum levels of antimicrobials. Adverse outcomes associated with antimicrobial levels outside the normal therapeutic range can be detected through prospective or retrospective audit.

- Morbidity and mortality reviews of nosocomial infections. Specific cases of adverse effects associated with antimicrobial use may be identified through regular departmental morbidity and mortality reviews.

- Infection control reports. Ongoing surveillance of nosocomial acquired infection, through the hospital infection control program, may identify certain adverse effects of antimicrobial use.

- Special audits. Special audits may be necessary to identify or further define additional potentially adverse effects not delineated by the aforementioned methods. This type of audit may be labour-intensive and should be used selectively.

\section{Analysis of data and formulation of an action plan}

The results of the surveillance activities should be reviewed on a monthly basis by the Antimicrobial Use Subcommittee. These data should be reviewed in light of pre-established guidelines of appropriate antimicrobial use and in collaboration with the medical staff.

Where practical, particularly in large hospitals, sharing information may be enhanced by the development of compatible computer networks between the various departments. Based on these interactions, an action plan for subsequent activity should be developed. This plan may include:

- Reporting (communication of surveillance findings)

The results of the surveillance should be made available to appropriate hospital committees and hospital administration. Through these bodies the information may be circulated to the rest of the hospital staff.

- Formulation of special audits Specific problems may have been identified which require further intensive investigation. This should be accomplished through the development of a focused database for surveillance activity and subsequent analysis of these activities.

\section{- Recommendations for intervention}

Based on surveillance activities, specific areas of drug misuse may be indicated which require intervention. These should be communicated to the hospital administration through the Pharmacy and Therapeutics Committee.

\section{Strategies to improve appropriate antimicrobial use}

These can be tailored according to the nature of the problem identified. Strategies for interventions include:

- educative and persuasive (re-education)

- facilitative

- restrictive (power).

As an integral part of the development of these strategies, a plan for analysis of outcome is required to ensure success.

Educative, persuasive and facilitative strategies should be aimed at the development of standardized guidelines for therapeutic regimens.

Educative and persuasive strategies

Interventions which have been used as educative and persuasive strategies to modify antimicrobial use include:

- Provision of teaching sessions as part of a continued medical education program. However, lectures have not been shown to be an effective means of modifying antimicrobial use.

- Provision of guidelines for antimicrobial use and information, including susceptibility patterns of hospital organisms. While the provision of such information would seem to be desirable, it has not been shown, by itself. to improve antimicrobial use.

- Audits with feedback to prescribing physicians. Audits which address specific problems of antimicrobial use have been shown to lead to modification of antimicrobial use in the short term, but changes are not sustained in the long term.

- Physician counselling (counter detailing). This is the provision of information about appropriate antimicrobial use on a physicianspecific basis. It has been shown to positively modify antimicrobial use, but it seems likely that continuous intervention must occur to maintain this modification.

- Concurrent review. Concurrent review is immediate review of a specific antimicrobial course of treatment initiated by the anti- 
microbial order. Feedback to the prescribing physician is provided if there is deviation from approved guidelines. This has been shown to have a positive impact on antimicrobial use.

Each institution will need to develop its own educational program. This will likely include a combination of the above methods. As a general rule however, to be effective, educational programs must be sustained, as well as being physician- and patient-specific.

\section{Facilitative strategies}

Facilitative strategies which have been employed to modify prescribing behaviour include clinical specialist consultations involving infectious diseases specialists, clinical microbiologists and clinical pharmacists. Facilitative strategies are frequently combined with educative, persuasive and restrictive strategies.

Restrictive (power) strategies. Various power strategies can be implemented to influence antimicrobial prescribing. These include restriction by:

- availability

- treatment regimen

- prescriber

- promotional activity.

Methods to achieve these goals include:

- Formulary restrictions and reserved antimicrobial program

A hospital drug formulary is a system which permits the evaluation and selection of therapeutically appropriate drugs and drug products (including antimicrobials) for use in the hospital. Drugs or drug products which are not considered effective or necessary should be designated as 'nonformulary' agents. When properly maintained, a drug formulary can lead to reduced drug costs, reduced inventories and more cost-effective prescribing habits of physicians. Decisions with respect to the formulary status of antimicrobials should be made through the Antimicrobial Use Subcommittee.

Only formulary antimicrobial agents should be available for routine use in the hospital. Nonformulary agents should only be used if suitable formulary alternatives are not available.

Certain antimicrobials may be available only on a reserved basis. These may be restricted for use in approved indications (ie, specific disease, patient group or organisms). Antimicrobial ordering forms may be used to facilitate this process. Prescribers should identify, when the drug is ordered, the approved indication for which the drug is intended. In some cases, the information provided (eg, prophylactic versus therapeutic intent) can be used to determine the automatic stop-order policy to be invoked.

- Selective reporting of susceptibility testing Susceptibility testing of microorganisms in a routine hospital diagnostic microbiology laboratory usually involves procedures performed in accordance with national standards. Microorganisms are tested against a predetermined panel of antimicrobial agents. These agents should reflect, as a general rule, those listed as part of the hospital drug formulary. The selection of antimicrobials for testing should be determined by the Pharmacy and Therapeutics Committee in conjunction with the Department of Microbiology.

All organisms tested should be reported as sensitive, intermediate or resistant. Reporting of minimal inhibitory concentration (MIC) data must be combined with some mechanism to ensure proper interpretation of the data.

To optimize antimicrobial use, a system of restricted reporting of susceptibility testing should be employed. Susceptibility information related to restricted or reserved agents should be reported only when requested by the clinician or where multi-resistant microorganisms have been isolated.

- Automatic stop policies Patients should be protected from indefinite, open-ended antimicrobial drug orders. Antimicrobial drugs which are not prescribed with a specified duration of therapy or number of doses indicated should be automatically discontinued after a reasonable period of time. To accomplish this, automatic stop-order policies should be established and approved by the Pharmacy and Therapeutics Committee (or equivalent) and Medical Advisory Committee (or equivalent). Policies should be documented in an appropriate hospital policy statement.

\section{- Therapeutic interchange programs}

Therapeutic interchange programs involve the substitution of one drug, drug regimen or route of administration for another. These programs represent a practical and effective mechanism to modify prescribing behaviour in the hospital. When the program involves 
an initial informational (persuasive) component, prescribers are encouraged to modify their prescribing behaviour before the interchange policy becomes effective. Two-stage interventions (persuasive and power strategies) are highly effective, and physician compliance is usually high.

- Antimicrobial order forms A form should be designed to ensure that information is provided by the prescriber regarding patient identification, drug, dosage regimen, route of administration and duration of therapy, as well as indication for use, suspected pathogen(s) and site(s) of infection. Using these preprinted forms, physicians should be obliged to categorize antimicrobial use as prophylactic or therapeutic. Depending upon the category, antimicrobials are automatically discontinued after a predefined time period or may not be provided if approved indications are not met.

- Required consultation and physician or service restriction

Orders for certain restricted drug(s) may require either formal consultation or counter signature by an individual or individuals designated by the Pharmacy and Therapeutics Committee. Only selected physicians or patient services (eg, intensive care unit) may be authorized by the Pharmacy and Therapeutics Committee to prescribe certain restricted antimicrobials.

- Institutional policy for pharmaceutical representative interactions

Interactions between pharmaceutical manufacturer representatives and hospital personnel should be conducted in a responsible and professional manner. The security and confidentiality of all hospital operations should be maintained at all times. The following policies are recommended:

- representatives should register their names, affiliation, contact person at the hospital and intent of visit with a designated department (eg, pharmacy) in the institution

- activities of the representative should be restricted to designated areas in the hospital. Detailing should not be permitted in patientcare areas
- educational or promotional materials should not be displayed or distributed in the institution without authorization

- drug product samples should be provided on a request basis only. Samples should be delivered to the Pharmacy Department and not distributed to patient-care areas

- representatives should be aware of, and identify during any discussions with hospital personnel, the formulary status of the drug product being detailed

- representatives violating hospital guidelines may lose hospital visitation privileges.

\section{GLOSSARY}

Usage: The term used to indicate the quality of drug use. The evaluation of drug usage is a multidisciplinary activity based on pre-established criteria/standards.

Utilization: The term used to indicate the quantity of drugs used. The collection and reporting of quantitative drug use data are pharmacy activities.

Use: The term used to indicate usage (quality) and utilization (quantity). The activities are multidisciplinary and involve collecting, analyzing and evaluating drug use data, combined with the actions needed to effect changes in prescribing practice.

Footnote: Further copies of this guideline together with annotated references can be obtained from Director, Bureau of Communicable Disease Epidemiology, Laboratory Centre for Disease Control, Health Protection Branch, Department of National Health and Welfare, Ottawa, Ontario K1A OL2. Also available are copies of other guidelines in the Infection Control series including: Guidelines for 1. Prevention of Intervascular Infections 2. Prevention of Surgical Wound Infections 3. Prevention of Nosocomial Pneumonia, 4. Prevention of Urinary Tract Infections, 5. Hospital Environmental Control (1988), Isolation and Precaution Techniques (1990), Long Term Care Facilities (1986), Occupational Health in Health Care Facilities (1986, under revision), Organization of Hospital Infection Control Programs (1985, under revision), Perinatal Care (1988). Guidelines for Infection Control Within a Correctional Environment have also been developed and have been published by the Correctional Service of Canada. 


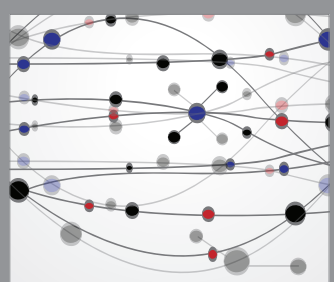

The Scientific World Journal
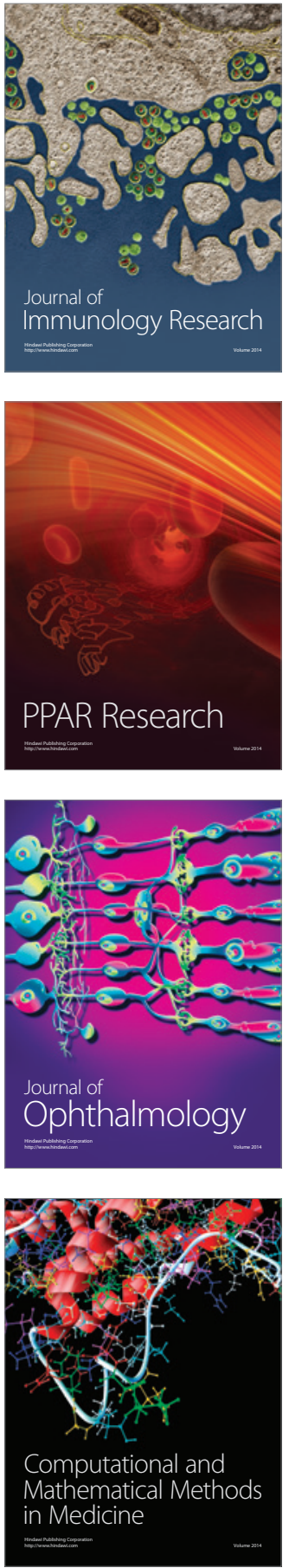

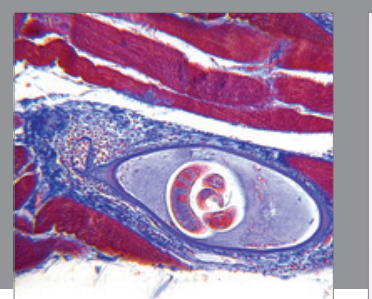

Gastroenterology Research and Practice

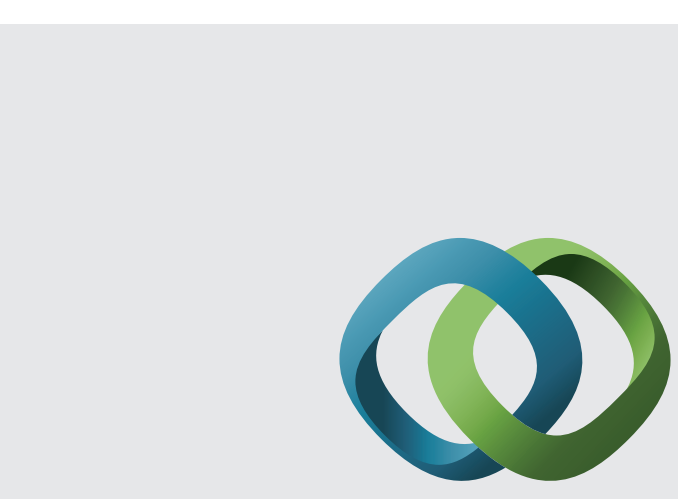

\section{Hindawi}

Submit your manuscripts at

http://www.hindawi.com
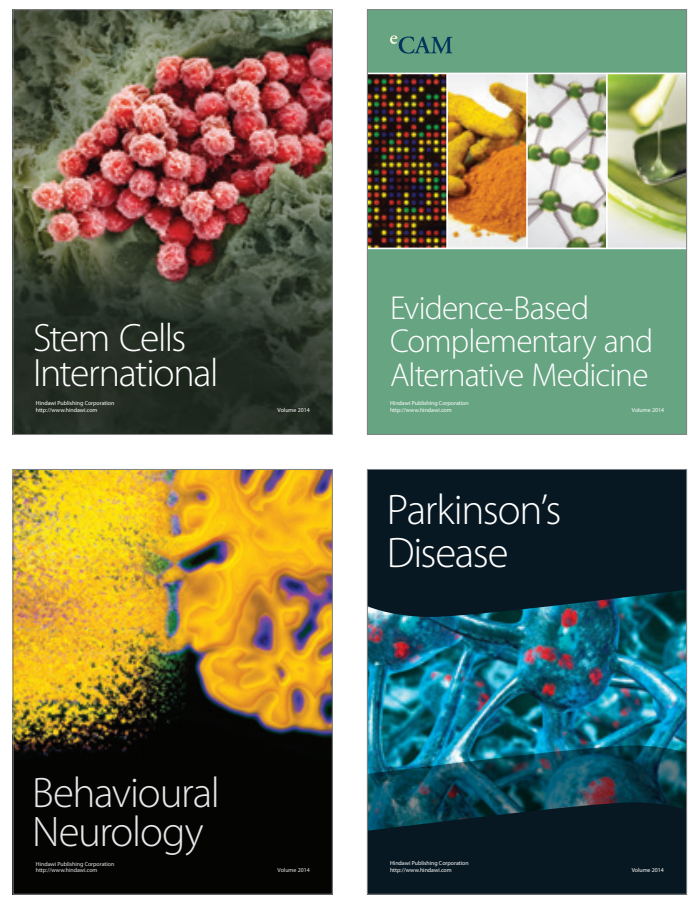
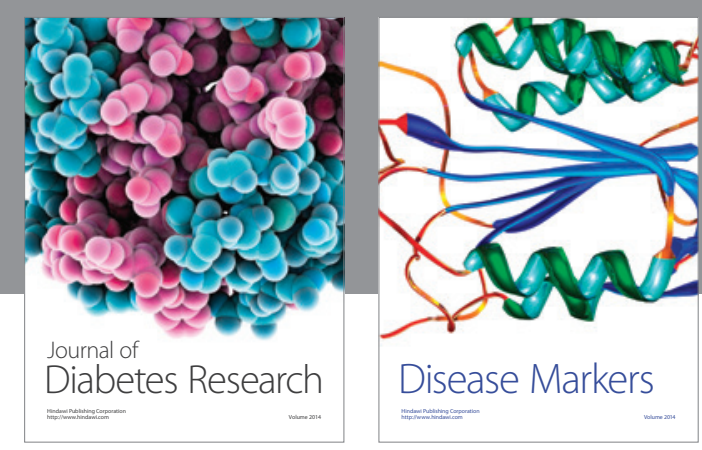

Disease Markers
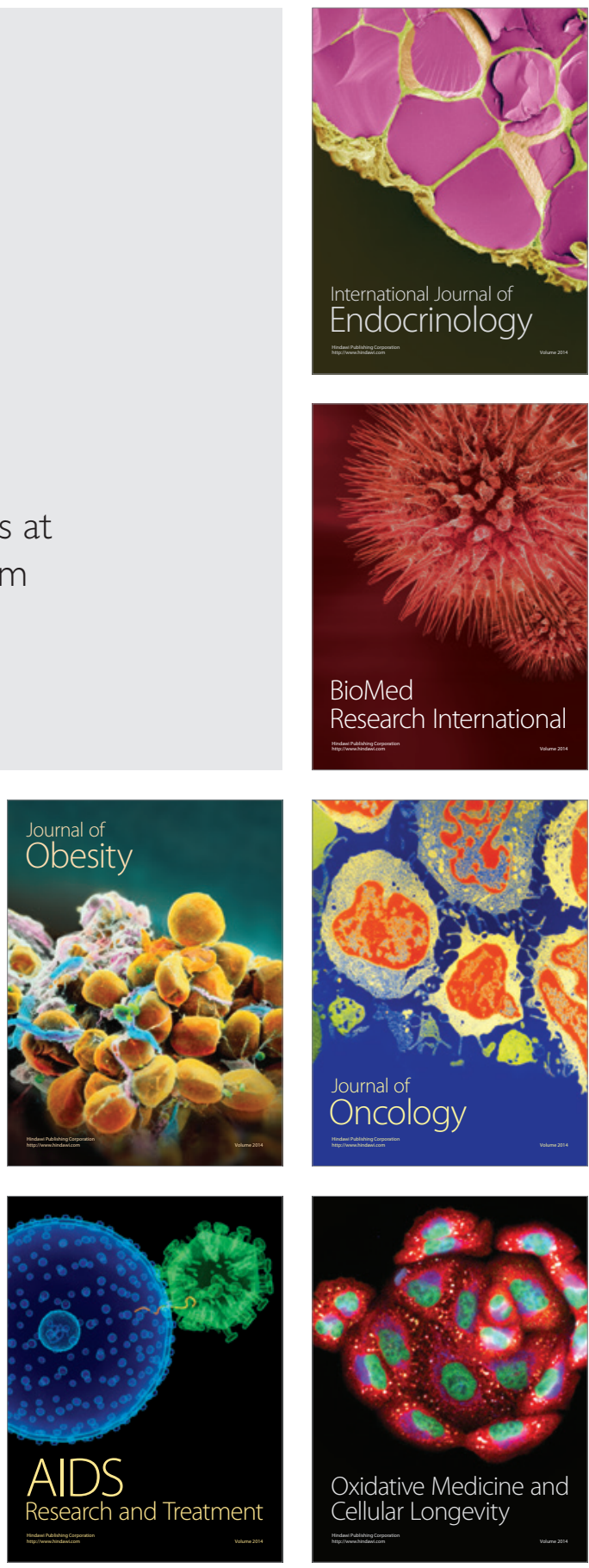